\title{
Papillary Thyroid Carcinoma: The First Case of Direct Tumor Extension into the Left Innominate Vein Managed with a Single Operative Approach
}

\author{
Douglas J Chung ${ }^{1}$, Diane Krieger ${ }^{2}$, Niberto Moreno ${ }^{3}$, Andrew Renshaw ${ }^{4}$, Rafael Alonso ${ }^{5}$, Robert Cava ${ }^{6}$, Mark Witkind ${ }^{7}$, \\ Robert Udelsman ${ }^{8}$
}

\begin{abstract}
Aim: The aim of this study is to report a case of papillary thyroid carcinoma (PTC) with direct intravascular extension into the left internal jugular vein, resulting in tumor thrombus into the left innominate vein.

Background: PTC is the most common of the four histological subtypes of thyroid malignancies, ${ }^{1}$ but PTC with vascular invasion into major blood vessels is rare. ${ }^{2}$ The incidence of PTC tumor thrombi was found to be $0.116 \%$ in one study investigating 7,754 thyroid surgical patients, and, of these patients with tumor thrombus, none extended more distal than the internal jugular vein. ${ }^{3}$ Koike et al. ${ }^{4}$ described a case of PTC invasion into the left innominate vein that was managed by a two-stage operative approach.

Case description: A 58-year-old male presented with a rapidly growing left thyroid mass. Fine needle aspiration cytology (FNAC) suggested PTC and surgical exploration confirmed tumor extension into the left internal jugular vein. Continued dissection revealed a large palpable intraluminal tumor thrombus extending below the clavicle into the mediastinum, necessitating median sternotomy.

Conclusion: Aggressive one-stage surgical resection resulted in successful en bloc extirpation of the tumor, with negative margins. Follow-up at 22 months postoperatively demonstrated no evidence of recurrence.

Clinical significance: This is the first case of PTC extension into the left innominate vein managed with one-stage surgical intervention with curative intent.

Keywords: Intravascular tumor extension, Innominate vein, Papillary thyroid carcinoma, Thyroid, Thyroid malignancy, Tumor thrombus.

World Journal of Endocrine Surgery (2019): 10.5005/jp-journals-10002-1249
\end{abstract}

\section{INTRODUCTION}

Papillary thyroid cancer (PTC) is the most common histological subtype of thyroid carcinoma, accounting for more than $85 \%$ of cases. ${ }^{1}$ While the prognosis is generally excellent (10-year survival rate exceeding 95\%), ${ }^{1}$ associated findings increase morbidity and mortality, including widespread metastatic disease and direct tumor extension into critical structures. PTC lymphatic spread is more common than hematogenous spread ${ }^{2}$ and the incidence of PTC cervical lymph node metastasis averages 60\%. ${ }^{1}$ Central and lateral nodal metastases are commonly seen, but with appropriate management, the prognosis is still excellent.

Direct tumor extension into large vessels is far less common, but may necessitate aggressive resection. One study demonstrated 9 thyroid surgery patients with intravascular tumor thrombus out of 7,754 followed in a 5-year study, an incidence rate of $0.116 \%$, but none extended distal to the internal jugular vein. ${ }^{3}$ Koike et al. ${ }^{4}$ described a two-stage operative approach in a patient with PTC in whom the thyroid was resected with the involvement of the left brachiocephalic vein.

\section{Case Report}

A 58-year-old male presented to his internist and was referred to an endocrinologist for a massive left thyroid nodule. The patient denied hoarseness, difficulty swallowing, difficulty breathing, or compressive symptoms. Physical examination revealed a mass in the left thyroid lobe with tracheal deviation from left to right. Laboratory data showed a normal serum TSH level of 2.43 (reference range: $0.40-4.50 \mathrm{mIU} / \mathrm{L}$ ).
${ }^{1}$ Department of Medical School, Herbert Wertheim College of Medicine, Florida International University, Miami, Florida, USA

${ }^{2}$ Department of Endocrinology, Endocrinology Associates, Miami, Florida, USA

${ }^{3}$ Department of Cardiothoracic Surgery, Baptist Health South Florida, Miami, Florida, USA

${ }^{4}$ Department of Pathology, Baptist Health South Florida, Miami, Florida, USA

${ }^{5}$ Institute of Endocrine Surgery, Miami Cancer Institute, Miami, Florida, USA

${ }^{6}$ Miami, Florida, USA

${ }^{7}$ Miami, Florida, USA

${ }^{8}$ Department of Medical School, Herbert Wertheim College of Medicine, Florida International University, Miami, Florida, USA; Institute of Endocrine Neoplasia, Miami Cancer Institute, Miami, Florida, USA

Corresponding Author: Robert Udelsman, Institute of Endocrine Neoplasia, Miami Cancer Institute, Miami, Florida, USA, Phone: +1786 596-2000, e-mail: RobertUd@baptisthealth.net

How to cite this article: Chung DJ, Krieger D, Moreno N, Renshaw A, Alonso R, Cava R, Witkind M, Udelsman R. Papillary Thyroid Carcinoma: The First Case of Direct Tumor Extension into the Left Innominate Vein Managed with a Single Operative Approach. World J Endoc Surg 2019;11(1):22-25.

Source of support: Nil

Conflict of interest: None

Thyroid ultrasound demonstrated a normal right thyroid lobe and isthmus, while the left thyroid lobe measured $9.5 \times 4.3 \times 4.5 \mathrm{~cm}$ 

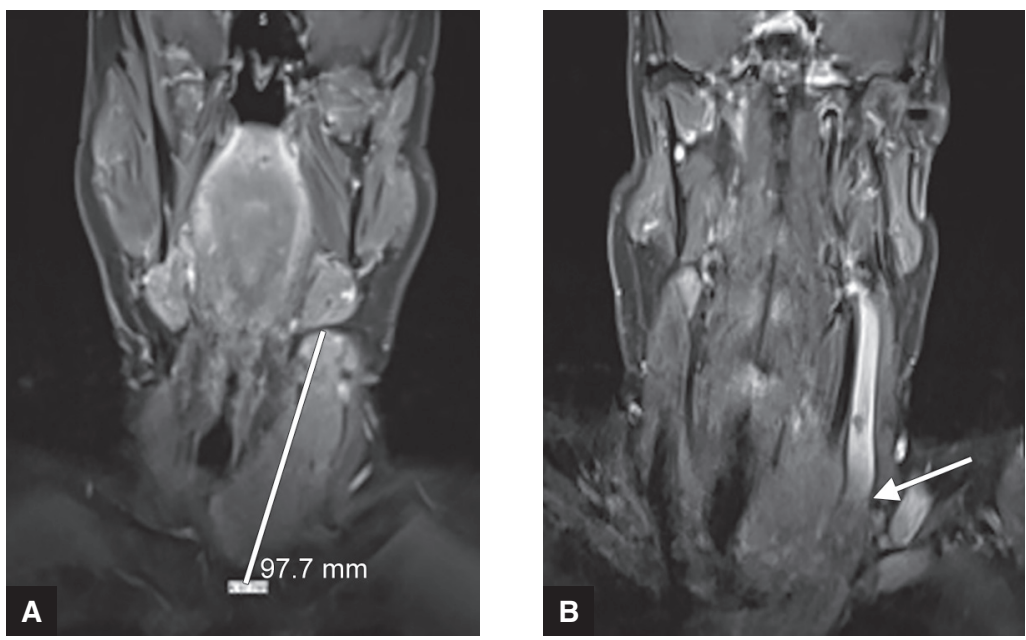

Figs $1 \mathrm{~A}$ and B: MRI of neck, coronal views. (A) $9.77 \mathrm{~cm}$ tumor replacing left thyroid lobe; (B) Direct tumor invasion (arrow) into left internal jugular vein

and was completely replaced by a heterogeneous hypoechoic mass highly suspicious for malignancy with no discernible normal thyroid tissue. The mass extended into the lumen of the left jugular vein, which demonstrated a vascular flow pattern highly suspicious for tumor thrombus with near complete occlusion. An FNAC demonstrated follicular cells with grooves and rare pseudoinclusions consistent with PTC.

The patient was seen by the endocrine surgeon and direct laryngoscopy demonstrated normal bilateral vocal cord function. Additionally, an MRI confirmed tumor extension into the left internal jugular vein, as shown in Figure 1. The patient is a speech pathologist and accepted the likelihood of aggressive extirpation and the probability of resection of his left recurrent laryngeal nerve.

During surgery, complete tumor resection required resection of strap muscles and the left recurrent laryngeal nerve due to gross tumor encasement. Uninvolved internal jugular vein superior to the tumor thrombus was identified, but inferiorly the tumor thrombus extended directly as far as could be palpated into the mediastinum toward the left innominate vein. Accordingly, a median sternotomy was performed for adequate resection.

The additional exposure revealed a fixed tumor thrombus extending directly into the left innominate vein, stopping proximal to the superior vena cava. The innominate vein and internal jugular vein were resected in continuity with the tumor. In addition, a left modified radical neck dissection was performed, resulting in complete tumor extirpation, as shown in the operative illustration (Fig. 2).

Pathology revealed a large infiltrative tumor arising in the left thyroid lobe (Fig. 3) and a tumor thrombus fixed to the wall of the left innominate vein (Fig. 4). Microscopic examination revealed PTC with tall cell features with direct extension into the left internal jugular and left innominate veins (Fig. 5). All lymph nodes were negative for metastasis and all margins were negative for tumor.

Postoperatively, the patient developed left arm swelling with thrombus in the left subclavian vein, which was treated with anticoagulation and elastic arm compression. Over 4 weeks, the swelling resolved and, with voice therapy, he resumed a normal speaking voice. Postoperative pretreatment radioactive iodine and positron emission tomography (PET) scans failed to demonstrate metastatic disease and he received $147 \mathrm{mCi}$ of iodine-131 (I-131) for radioablation following thyrogen stimulation and his post-ablative scan failed to demonstrate residual disease. Six months after surgery, the patient had a normal ultrasound without evidence of metastatic adenopathy or cervical recurrence. Follow-up at 22 months postoperatively demonstrated a normal postoperative exam, a thyroglobulin level of $0.6 \mathrm{ng} / \mathrm{mL}$ with negative antithyroglobulin antibodies, a negative CT scan, and an I-131 scan negative for evidence of recurrence.

\section{Discussion}

PTC is the most common subtype of thyroid malignancy, accounting for more than $85 \%$ of cases. ${ }^{1}$ While the prognosis of PTC is excellent (10-year survival rate exceeding 95\%), ${ }^{1}$ PTC invasion into the great veins is extremely rare. ${ }^{2}$ Kobayashi et al.' $s^{3}$ data on 7,754 thyroid surgery patients treated at the Kuma Hospital in Japan between 2004 and 2009 demonstrated only 9 patients with major intravascular tumor thrombi, about $0.116 \%$. Of these, six had invasion in the internal jugular vein and three with invasion in a thyroid vein, ${ }^{3}$ but no patients had innominate vein involvement. To the best of our knowledge, there have been around 10 cases of innominate vein or superior vena cava tumor thrombus by other subtypes of thyroid carcinoma (anaplastic or follicular). Koike et al. ${ }^{4}$ described a patient with poorly differentiated PTC and involvement of the brachiocephalic vein in whom a two-stage operative approach was performed.

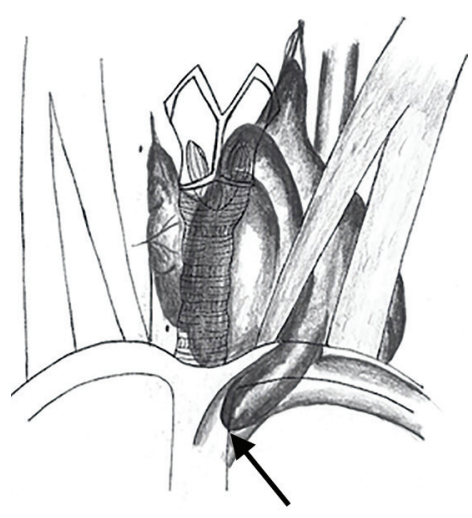

Fig. 2: Operative illustration demonstrating large infiltrative tumor arising in the left thyroid lobe with direct extension into left internal jugular vein and tumor thrombus extending into left innominate vein (arrow) 


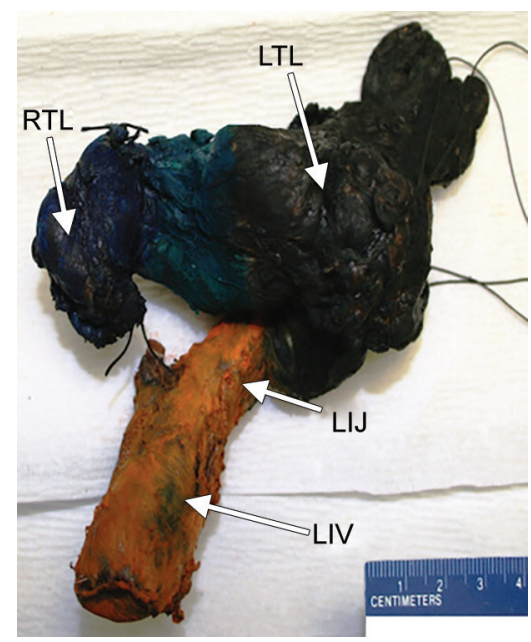

Fig. 3: Gross resected specimen. The thyroid and strap muscles are inked in blue and the left internal jugular and innominate veins are inked in orange. RTL, right thyroid lobe; LTL, left thyroid lobe; LIJ, left internal jugular vein; LIV, left innominate vein

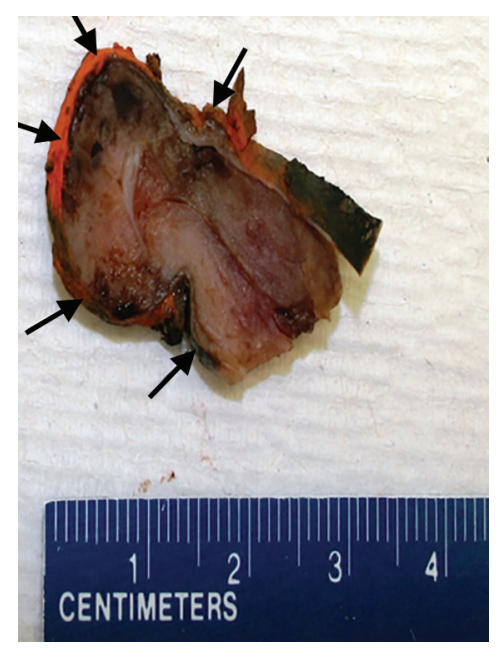

Fig. 4: Gross image of tumor thrombus inside the lumen of the left innominate vein. The tumor-endothelial border is shown by the arrows

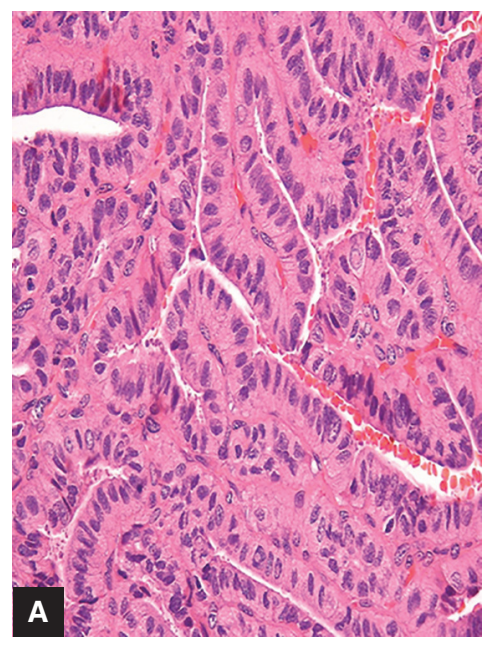

The tumor could not be resected during initial operation because the cervical approach was insufficient to completely excise the tumor. Therefore, a second operation with median sternotomy was performed which found that the tumor was extending into the left innominate vein and floating in the superior vena cava. En bloc resection was completed and the patient remained alive 8 months postoperatively at the time of publication. ${ }^{4}$

Demonstration of PTC tumor extension into the great veins can be observed with thyroid ultrasound, computed tomography (CT), or magnetic resonance imaging (MRI) scans. ${ }^{5}$ While we were aware of the left internal jugular vein involvement from preoperative ultrasound and MRI scans, PTC tumor thrombus into the left innominate vein was revealed during surgical exploration, prompting median sternotomy for adequate tumor resection in continuity.

Aggressive surgical resection produced negative gross and microscopic margins. Resection of the left innominate vein was required to achieve these margins and is well tolerated. A study by Arthur et al. ${ }^{6}$ demonstrated that division and ligation of the left innominate vein is safe for patients undergoing mediastinal tumor excisions. Postoperatively, our patient developed left arm swelling with thrombus in the left subclavian vein and was treated with anticoagulants and elastic arm compression. McPhee et al. ${ }^{6}$ demonstrated that these findings are part of the normal recovery process for left innominate vein resection and improve with conservative management as collateral vessels form. At 1-month postoperative follow-up, the patient's swelling completely resolved.

Pulmonary metastases were considered due to the tumor's location within a major vessel and the possibility that cancer cells had been released, traveling through the right atrium, seeding the lungs. We evaluated pulmonary metastasis with preradioactive iodine imaging (RAI) treatment with thyrogen-stimulated PET and RAI scanning, which were negative for pulmonary metastasis. Additionally, $85 \%$ of PTC respond to I- 131 and efficacy is improved by early postoperative treatment when there is only a microscopic tumor burden. It is also possible that the patient did not have metastasis, in which case I-131 will likely have prophylactic value.

Stickel et al. ${ }^{7}$ described the first anaplastic thyroid carcinoma (ATC) with tumor extension into the great vessels by postmortem exam. Autopsy revealed that ATC of the left thyroid lobe was extending down the internal jugular vein into the right atrium. The right ventricle was lined with metastases and the lungs

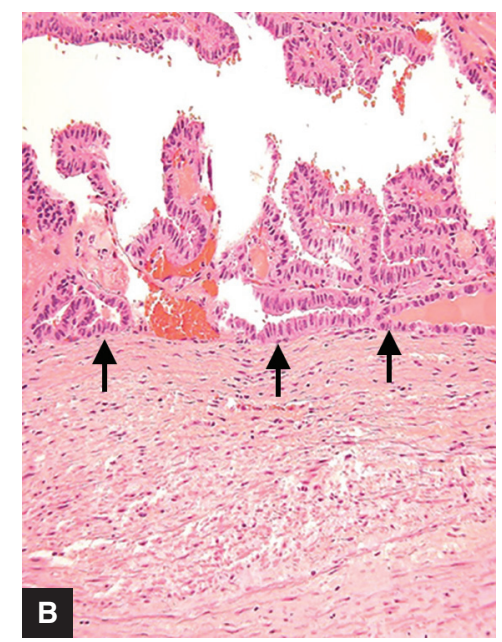

Figs 5A and B: Microscopic demonstration of tumor stained by hematoxylin and eosin under low power; (A) Papillary thyroid cancer of the thyroid with tall cell features; (B) Tumor found in the left innominate vein adhered to the lining of the left innominate vein as shown by the arrows 
demonstrated multiple pulmonary infarctions from tumor and non-tumor thrombi. ${ }^{7}$ ATC is much less common than PTC and there have been other cases where ATC intravascular extensions reached the right atrium and the ATC appeared to have arisen from the transformation of PTC. ${ }^{8}$

Despite the lack of established management guidelines due to the rarity of PTC vascular thrombus, Al-Jarrah et al.'s $\mathrm{s}^{9}$ report supports aggressive surgical treatment due to the relatively good prognosis of PTC and evidence that their patient's tumor was not attached to the vascular wall, making thrombus extraction and vascular repair feasible. Our patient received aggressive surgical treatment with negative margins by pathology and has done well postoperatively. As demonstrated in Figure 5B, because his PTC had been attached to the vascular endothelium, thrombectomy would not have been a judicious option.

In summary, this case represents the first report of PTC with direct extension into the internal jugular vein with tumor thrombus extending into the left innominate vein managed in a single operative approach with curative intent. Aggressive en bloc onestage surgical resection resulted in negative margins, and, at nearly 2-year follow-up, the patient is disease free.

\section{Acknowledgments}

Authors are indebted to the patient for undergoing aggressive treatment for a rare presentation of thyroid cancer.

\section{References}

1. Liang K, He L, et al. Risk Factors of Central Lymph Node Metastasis in cNO Papillary Thyroid Carcinoma: A Study of 529 Patients. Med Sci Monit 2014;20:807-811. DOI: 10.12659/MSM.890182.

2. Dikici AS, Yıldırım O, et al. A Rare Complication of the Thyroid Malignancies: Jugular Vein Invasion. Pol J Radiol 2015;80:360-363. DOI: 10.12659/PJR.894057.

3. Kobayashi K, Hirokawa M, et al. Tumor Thrombus of Thyroid Malignancies in Veins: Importance of Detection by Ultrasonography. Thyroid. https://www.ncbi.nlm.nih.gov/pubmed/21476893. Published May 2011. Accessed April 14, 2018.

4. Koike E, Yamashita H, et al. Brachiocephalic Vein Thrombus of Papillary Thyroid Cancer: Report of a Case. Surg Today 2002;32(1):59-62. DOI: 10.1007/s595-002-8114-7.

5. Marcy PY, Thariat J, et al. Jugular Vein Invasion Diagnosis and Prognosis in Thyroid Carcinomas. Pol J Radiol 2016;81:268-269. DOI: 10.12659/PJR.896757.

6. McPhee A, Shaikhrezai K, et al. Is it safe to divide and ligate the left innominate vein in complex cardiothoracic surgeries? Interact Cardiovasc Thorac Surg 2013;17(3):560-563. DOI: 10.1093/icvts/ivt244.

7. Stickel J, Mayer F, et al. Ventricular Thrombus and Thrombocytopenia As First Presentation of Anaplastic Thyroid Carcinoma. J Clin Oncol 2013;31(12):e194-e196. DOI: 10.1200/JCO.2012.43.4894.

8. Sugimoto $\mathrm{S}$, Doihara $\mathrm{H}$, et al. Intraatrial Extension of Thyroid Cancer: A Case Report. Acta Med Okayama 2006;60:135-140. DOI: 10.18926/AMO/30734. https://www.ncbi.nlm.nih.gov/pubmed/ 16680191. Accessed April 11, 2018.

9. Al-Jarrah Q, Abou-Foul AK, et al. Intravascular Extension of Papillary Thyroid Carcinoma to the Internal Jugular Vein: A Case Report. Int J Surg Case Rep 2014;5(8):551-553. DOI: 10.1016/j.ijscr.2014.06.005. 